\title{
Scientific Collaboration among Science and Engineering Universities ---Network Visualization of Co-authored papers
}

\author{
$\mathrm{Na} \mathrm{Li}$
}

South China University of Technology Library, Guangzhou, Guangdong, China

\begin{abstract}
To reveal the current status of scientific cooperation among Science and Engineering Universities, the cooperation frequency matrix is found basing on the datum of co-authored papers of Science and Engineering Universities in 2013 from the SCIE (Science Citation Index Expanded) database. The investigated universities compose of thirty six top100 ranking Science and Engineering Universities in china. The cooperation network is visualized by Ucinet and Netdraw. Results show both the number of papers published and the number of co-authored papers in Tsinghua University are more than other Science and Engineering University. Regional proximity is the main factors to affect cooperative strength among Science and Engineering Universities. The cooperation network visualization is a feasible method for scientific cooperation research.
\end{abstract}

Keywords—science and engineering university, co-authored papers, social network analysis, visualization

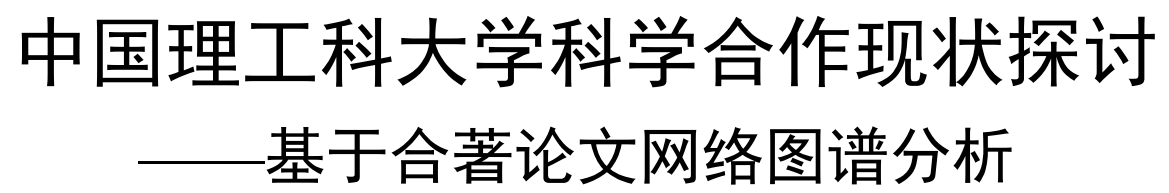

李娜

华南理工大学图书馆, 广州, 广东, 中国

摘 要 为了从宏观上把握中国理工科大学间科学合作现状, 选择中国大学排行榜百强中的 36 所理工科大学为研究对象, 基于 SCIE 数据库检索出 2013 年理工科大学之间的论文合著情况, 并建立了论文合著频率矩阵。然后借助 Ucinet 和 Netdraw 软件绘制理工 科大学间科学合作的可视化网络图谱。研究发现: 清华大学无论是论文发表总量还是合作总量都高于其他理工科大学, 并在网络图谱 中与其他大学形成很强的合作关系; 地域相近性是影响理工科大学间科学合作关系强弱的主要因素; 理工科大学间科学合作可视化网 络图谱分析是揭示科技创新合作现状的可行手段。

关键词 理工科大学，合著论文，社会网络分析，可视化

科学合作已经成为科学研究中的一个重要方面, 这源 于科学发展的复杂性、技术的飞速变化和知识的动态增长 以及高度发展的专门知识和技能, 科学合作是实现研究资 源共享、减低科研难度和学者获得学术声誉的重要形式[1]。 美国科学社会学家朱克曼(Zuckerman)在 20 世纪 70 年代通 过一项研究发现, 在诺贝尔奖设立的头 25 年中, 合作获奖 者占 $41 \%$; 第二个 25 年达到 $65 \%$; 第三个 25 年则已高达 $79 \%[2]$ 。所以, 分析科学合作现状及规律正成为科学计量
学者关注的热点问题。科学合作以其主体的不同显现为不 同层次的合作形式。大学是科学研究活动的重要主体之一, 在科学合作中扮演着重要角色, 有关大学科学合作问题在 我国学术界也深受学者关注, 研究最多的是大学与企业间 的合作, 大学之间的合作是研究大学科学合作的另一个视 角[3]。如果将合作大学视为一个小团体, 合作倾向性反映 的则是小团体成员间的疏密关系。

2014 年 3 月 26 日, 中国校友会网最新编制完成《2014

中央高校基本科研业务费资助项目（项目编号：2014GM07） 
中国大学评价研究报告》, 报告显示, 在中国校友会网 2014 中国大学排行榜 100 强中, 理工类院校有 36 所[4]。为了解 中国理工科大学之间的科学合作现状, 本文选择跻身中国 大学排行榜百强的 36 所理工科大学作为样本, 构建理工科 大学科学合作小团体, 基于大学与大学间论文合著情况, 绘制理工科大学间科学合作图谱, 从宏观和定量的角度, 可视化地展现理工科大学科学合作现状, 为今后完善我国 理工科大学发展战略及科研评估政策提供参考建议。

\section{1 数据与方法}

数据来源于 Web of Science 中的 Science Citation Index Expanded (简称 SCIE) 数据库。为了反映中国理工科大学 间科学合作的最新现状, 将数据检索的时间范围限定在 2013 年; 选择地址字段分别输入大学名称英文缩写形式进 行检索; 为提高文献数据分析的精确性, 选择 Article 类型 文献。检索时间是 2014 年 9 月 24 日到 2014 年 9 月 26 日。 首先, 仅选择一所大学作为检索单位进行检索, 共计检索 36 次, 分别得到 36 所理工科大学 2013 年发表的论文总数; 然后, 再选择两所大学作为检索单位以“and”逻辑关系进行 检索, 共计进行了 612 次交叉检索, 得到每所大学与其他 大学 2013 年合作发表的论文数量。

以往的科学合作分析通常采用统计分析方法, 通常意 义上的各种统计分析方法多数不能用来分析关系数据。社
会网络分析恰恰研究的是关系数据。社会网络分析由于引 入数学图论和计算机技术为手段而不断成熟, 给社会学带 来了新的概念和理论, 使得社会学对社会结构的研究更加 深入, 一些模型和方法也被广泛应用到社会学的许多领域 [5]。社会网络分析是一种新的社会科学研究范式, 关注行 动者之间的“关系”和整体网络的结构, 在本体论、认识论、 方法论、以及具体的研究方法上都有其独到之处[6]。本文 首先基于 SCIE 检索数据, 描绘出中国理工科大学科学研究 水平以及理工科大学间科学合作的总体情况。然后采用社 会网络分析方法, 并借助广为流行 Ucinet 和 Netdraw 工具 软件, 以理工科大学为节点, 对理工科大学间科学合作的 网络结构进行可视化分析。

\section{2 结果与分析}

\section{1 合作频次与网络图谱}

根据检索得到理工科大学之间合作发表的论文数量, 建立一个全完对称的三角形矩阵, 详见表 1 。将表 1 的矩 阵导入 Ucinet 软件, 再用 Netdraw 工具绘制出理工科大学 间论文合作的网络图谱, 详见图 1 和图 2。图中节点代表 了各个理工科大学, 节点的大小表示程度中心性, 连线的 粗细表示两所大学间合作频次情况。

表 136 所理工科大学 2013 年论文合作频次矩阵（片断）

\begin{tabular}{|c|c|c|c|c|c|c|c|c|c|c|c|}
\hline 合作论文数量 & $\begin{array}{l}\text { 华中 } \\
\text { 科技 } \\
\text { 大学 }\end{array}$ & $\begin{array}{c}\text { 哈尔 } \\
\text { 滨工 } \\
\text { 业大 } \\
\text { 学 }\end{array}$ & $\begin{array}{l}\text { 中国 } \\
\text { 科学 } \\
\text { 技术 } \\
\text { 大学 }\end{array}$ & $\begin{array}{l}\text { 天津 } \\
\text { 大学 }\end{array}$ & $\begin{array}{l}\text { 同济 } \\
\text { 大学 }\end{array}$ & $\begin{array}{l}\text { 大连 } \\
\text { 理工 } \\
\text { 大学 }\end{array}$ & $\begin{array}{l}\text { 华南 } \\
\text { 理工 } \\
\text { 大学 }\end{array}$ & $\begin{array}{l}\text { 东北 } \\
\text { 大学 }\end{array}$ & $\begin{array}{l}\text { 电子 } \\
\text { 科技 } \\
\text { 大学 }\end{array}$ & $\begin{array}{l}\text { 北京 } \\
\text { 航空 } \\
\text { 航天 } \\
\text { 大学 }\end{array}$ & $\begin{array}{l}\text { 华东 } \\
\text { 理工 } \\
\text { 大学 }\end{array}$ \\
\hline 清华大学 & 36 & 47 & 80 & 52 & 19 & 17 & 11 & 21 & 14 & 55 & 7 \\
\hline 华中科技大学 & & 8 & 14 & 16 & 15 & 12 & 5 & 1 & 10 & 10 & 0 \\
\hline 哈尔滨工业大学 & & & 9 & 19 & 23 & 65 & 8 & 6 & 7 & 11 & 9 \\
\hline 中国科学技术大学 & & & & 6 & 16 & 5 & 17 & 29 & 7 & 23 & 2 \\
\hline 天津大学 & & & & & 6 & 20 & 7 & 2 & 5 & 13 & 4 \\
\hline 同济大学 & & & & & & 8 & 5 & 1 & 2 & 4 & 18 \\
\hline 大连理工大学 & & & & & & & 16 & 22 & 3 & 10 & 9 \\
\hline 华南理工大学 & & & & & & & & 1 & 5 & 3 & 4 \\
\hline 东北大学 & & & & & & & & & 0 & 3 & 2 \\
\hline 电子科技大学 & & & & & & & & & & 2 & 1 \\
\hline 北京航空航天大学 & & & & & & & & & & & 0 \\
\hline
\end{tabular}




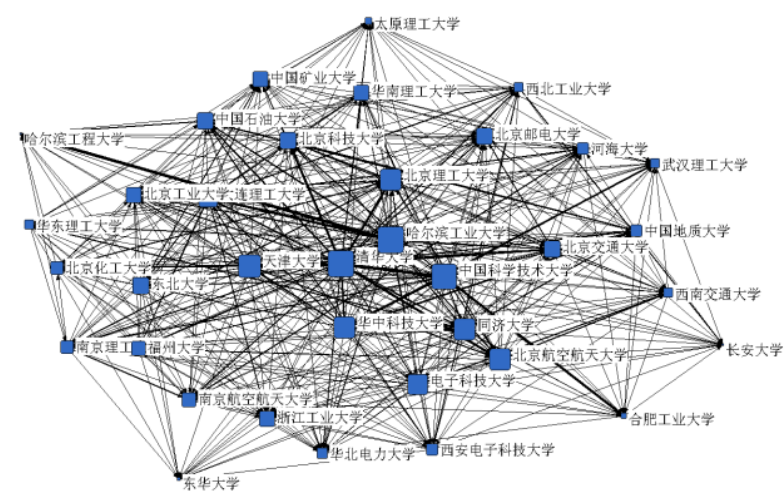

图 136 所理工科大学论文合作网络图谱（度数中心性）

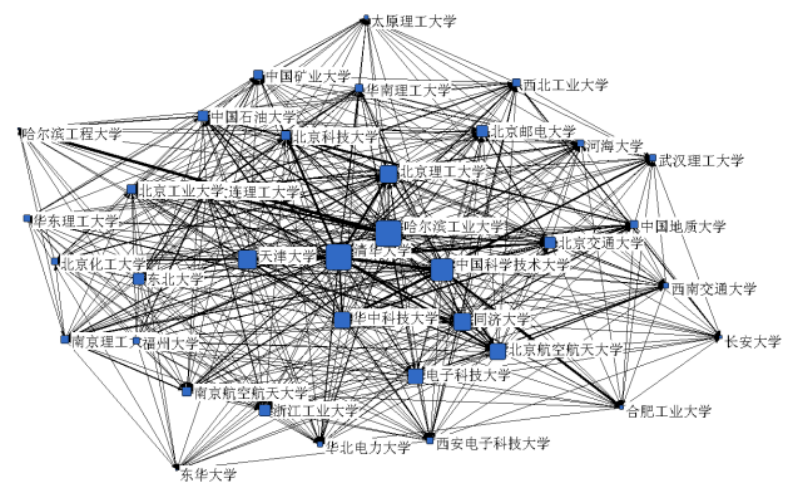

图 236 所理工科大学论文合作网络图谱（中间中心性）

简单地说, 如果一个点与其它许多点直接相连, 我们 就说该点具有较高的度数中心性, 在这个网络中居于中心 地位, 从而拥有较大的权力[6]。从图 1 节点的大小可以看 出, 哈尔滨工业大学、清华大学、中国科学技术大学、天 津大学、华中科技大学、同济大学、北京航空航天大学、 北京理工大学等八所大学的度数中心性比较高, 说明这几
所大学处于网络的重要地位, 在该网络中拥有较大影响力。 中间中心性是测量行动者对资源的控制的程度, 如果 一个点处于许多其它点对的捷径（最短的途径）上, 可以 说该点具有较高的中间中心性[6]。在此意义上说, 该点起 到沟通各个他者的桥梁作用。从图 2 节点的大小可以看出, 哈尔滨工业大学、清华大学、中国科学技术大学、天津大 学、同济大学、北京理工大学、华中科技大学、北京航空 航天大学等八所大学的中间中心性比较高, 由直觉可知, 这几所大学在网络路径中居于重要的地位, 具有控制其他 大学之间交往的能力。另外, 虽然点度中心性较高的八所 大学与中间中心性较高的八所大学相同, 但其强度顺序不 同, 说明在社交网络中处于核心地位的大学, 并不一定对 资源控制能力最强, 反之, 在社交网络中资源控制能力最 强的大学, 并不一定处于核心地位。

\section{2 合作倾向性与网络图谱}

在用 Netdraw 绘制理工科大学合作网络图谱时, 可以 设置不同的阈值来简化合作网络结构。通过过滤掉小于阈 值的数据, 减少网络节点和连线的数量, 使整个网络结构 变得清晰。但是设置阈值的大小非常关键, 如果阈值设置 过小，对网络结构改善不明显；如果阈值设置过大，将会 有许多理工科大学在网络结构中无法体现。所以必须选择 合适的阈值, 既能简化网络结构, 又能保留主要大学的数 量。最后, 通过观察简化的网络图谱, 分析出各理工科大 学间科学合作倾向性。

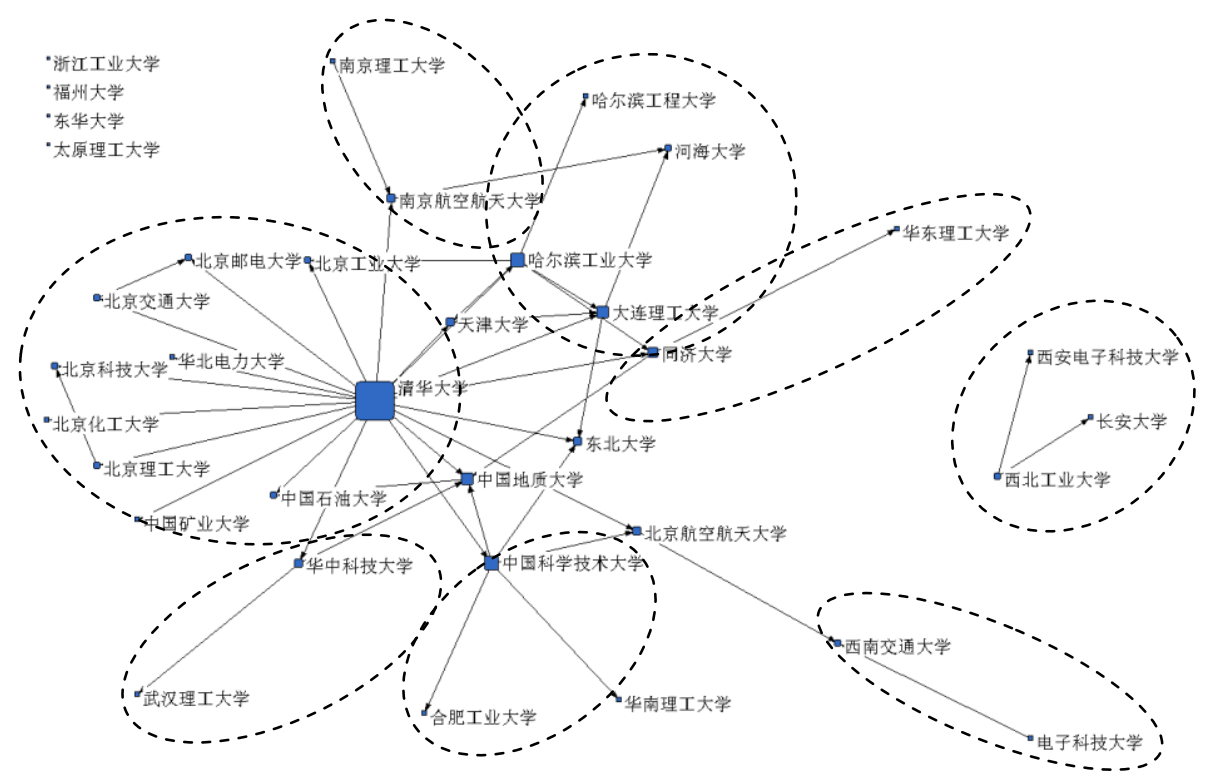

图 336 所理工科大学论文合作网络图谱（阈值=16） 
从图 3 中可以看出, 36 所理工科大学间最强的合作关 系几乎都发生在同一个城市或同一个地区, 由此说明理工 科大学论文合作规律主要是地域相近性, 在图 3 中用虚线 框将能体现地域相近的子网络标识出来。由清华大学、北 京理工大学、北京科技大学、北京化工大学、北京交通大 学、北京邮电大学、北京工业大学、中国地质大学组成北 京地区科学合作子网络; 由西安电子科技大学、长安大学、 西北工业大学组成西安地区科学合作子网络; 由哈尔滨工 业大学、哈尔滨工程大学、大连理工大学组成东北地区科 学合作子网络; 由南京理工大学、南京航空航天大学、河 海大学组成南京地区科学合作子网络; 由同济大学、华东 理工大学组成上海地区科学合作子网络; 由西南交通大学、 电子科技大学组成成都地区科学合作子网络; 由中国科学 技术大学、合肥工业大学组成合肥地区科学合作子网络; 华中科技大学、武汉理工大学组成武汉地区科学合作子网 络。最终得出这样的结论: 地域相近性是影响中国理工科 大学间科学论文合作关系强弱的最大因素。像北京、西安、 东北地区科学论文合作存在非常明显的地域相近性。另外, 由于阈值设为 16 , 表示理工科大学间论文合作频次低于 16 的弱连接将被忽略掉, 如浙江工业大学、福洲大学、东华 大学、太原理工大学, 由于这几所大学与其他大学单项合 作频次小于 16 , 所以在合作网络图谱中未能得到体现。

\section{3 结论}

科技论文是科技工作者进行科研活动的主要成果形 式, 也是科学计量学和科学知识网络图谱的主要研究对象 [7]。本次研究以跻身中国大学排行榜百强的 36 所理工科大 学作为样本, 通过 SCIE 数据库检索 2013 年理工科大学间 论文合作情况, 建立了论文合作频率矩阵, 用 Netdraw 绘 制了社会网络分析图谱。通过可视化网络图谱和相关统计 数据分析, 从宏观上把握中国理工科大学间科技合作现状。 本次研究的主要发现是:

(1) 理工科大学间的科学论文合作网络从整体上看是 一个联系比较广泛的网络, 各个理工科大学之间或多或少 都有论文合作上的联系。哈尔滨工业大学、清华大学、中 国科学技术大学、天津大学、华中科技大学、同济大学、 北京航空航天大学、北京理工大学这八所大学在整个网络 图谱中不仅居于核心地位, 而且也是对资源的控制程度最 强的理工科大学。

(2) 理工科大学间合作的活跃程度与其自身的学术影 响力和创造力有一定的关系。例如清华大学, 无论是科学 论文的发文总量和合作总量明显高于其他理工科大学, 在 网络图谱中与其他大学形成很强的合作关系。例如浙江工 业大学、福洲大学、东华大学、太原理工大学在网络图谱
中表现不够活跃, 处于边缘地位, 这些大学亟需加强科学 合作, 推动科学技术的突破和创新。

(3) 地域相近性是影响理工科大学间科学合作关系强 弱的主要因素。在最小距离范围内寻找合作对象, 无论是 人员交流还是资源共享都是最便利的, 符合最小投入、最 大收益的经济学规律。

(4) 未发现学科相似性对理工科大学间科学合作关系 强弱有明显的影响, 这是因为本次研究是以理工科大学为 分析样本, 这些大学本身就具有理工科的相似性, 所以在 网络图谱中未见到明显的学科相似性也是合理的。

本次研究结果表明, 理工科大学间科学合作可视化网 络图谱分析是提示科技创新合作现状的可行手段。由于本 次研究仅以合著的 SCIE 论文为分析对象, 未选择合作专 利、合作科技成果、中文数据库合著论文等进行多角度综 合分析, 而且数据仅限于 2013 年, 可能会存在数据不够全 面的问题; 又由于理工科大学样本数量选取有限, 对理工 科大学间科学合作强度统计很难体现整体性, 这些问题有 待在后续工作中进一步探讨。

\section{参考文献(References)}

[1] Hara N, Solomon P, Kim SL. An emerging view of scientific collaboration: Scientists' perspectives on collaboration and factors that impact collaboration. JOURNAL OF THE AMERICAN SOCIETY FOR INFORMATION SCIENCE AND TECHNOLOGY, 2003, 54(10): 952-965.

[2] Glanzel W. Gzerwon H. A new methodological approach to bibliography coupling and its application to national, regional and institutional level. Scientometrics, 1996(2): 195-221.

[3] LIANG Liming, SHA Dechun. Strong Geographical Preferences in Scientific Collaboration between 985 Project Universities. Science of science and management of science and technology. 2008, 11: 112-116.

[4] 2014 China Polytechnic University Rankings. (2014-03-28) [2014-09-24].

http://edu.sina.com.cn/gaokao/2014-03-28/1020413570.shtml.

[5] WU Dong. Research on complex networks and its significance. Study of Philosophy.2004, 08: 58-63.

[6] LIU Jun. The whole network analysis notes. Gezhi Publishing House.2009.

[7] SU Yifan, ZHU Shaoqiang, XIE Weihong. Scientific Research Cooperation among Universities within Guangzhou City: Knowledge Visualization by Co-authorship Data in 2012. Science and technology management research.2014, 06: 90-95. 